\title{
THE TIME COURSE OF THE EFFECTS OF CENTRAL AND PERIPHERAL CUES ON VISUAL PROCESSING: AN EVENT-RELATED POTENTIALS STUDY
}

\author{
Authors: Sonia Doallo a , Laura Lorenzo-López ${ }^{a}$, Carmen Vizosoa ${ }^{a}$, Socorro Rodríguez Holguín a , Elena \\ Amenedo $^{\mathrm{a}}$, Salvador Bará ${ }^{\mathrm{b}}$, Fernando Cadaveira ${ }^{\mathrm{a}}$
}

This is the peer reviewed version of the following article: Doallo S; Lorenzo-López L; Vizoso C, Rodríguez Holguín S, Amenedo E, Bará S, Cadaveira F. (2004). The time course of the effects of central and peripheral cues on visual processing: an event-related potentials study. Clinical Neurophysiology 115, 199-210, doi:10.1016/S1388-2457(03)00317-1. This article may be used for non-commercial purposes in accordance with Elsevier Terms and Conditions for Use of SelfArchived Versions 
The time course of the effects of central and peripheral cues on visual processing: an event-related potentials study

Authors: Sonia Doallo ${ }^{\text {a }}$, Laura Lorenzo-López ${ }^{\text {a }}$, Carmen Vizoso ${ }^{a}$, Socorro Rodríguez Holguína ${ }^{\text {a }}$ Elena Amenedo $^{a}$, Salvador Baráb, Fernando Cadaveira ${ }^{a}$

${ }^{a}$ Departamento de Psicoloxía Clínica e Psicobioloxía, Facultade de Psicoloxía, Universidade de Santiago de Compostela, Campus Universitario Sur, E-15782, Santiago de Compostela, Galicia, Spain

${ }^{b}$ Departamento de Física Aplicada, Facultade de Física, Universidade de Santiago de Compostela, Campus Universitario Sur, E-15782, Santiago de Compostela, Galicia, Spain

Corresponding author: Sonia Doallo Tel.: +34 981563100 ext. 13916; fax: + 34981521 581. Email address: sdoallo@usc.es

1 


\begin{abstract}
Objective: The varying results of visual event-related potential (ERP) studies of central and peripheral cueing suggest that these types of cue may modulate stimuli processing with different time courses. The aim of this study was to investigate differences in the time course of facilitatory effects on the visual processing induced by peripheral and central cues.
\end{abstract}

Methods: ERPs were recorded for visual target stimuli that were preceded by informative-central, informative-peripheral or uninformative-peripheral cues with stimulus onset asynchronies (SOAs) of $100,300,500$ or $700 \mathrm{~ms}$.

Results: Validly cued stimuli elicited an enhanced P1 component with peripheral cueing at $100 \mathrm{~ms}$ SOA. P1 amplitude in valid trials was reduced at 300, 500 and $700 \mathrm{~ms}$ SOAs with uninformativeperipheral cueing, but only at $500 \mathrm{~ms}$ SOA with informative-peripheral cueing. With informativecentral cueing, there was no validity effect on P1.

Conclusions: These results suggest that the automatic attraction of attention by a peripheral cue results in improved sensory processing at the cued location. This facilitation is replaced by an inhibitory effect when SOA increases, although cue informativeness may modulate this effect. Central cueing does not affect sensory processing at the P1 level.

\title{
Keywords
}

Visuospatial attention; Central cues; Peripheral cues; P1; Event-related potentials 


\section{Introduction}

It has been well established that when attention is directed toward locations within the visual field, stimuli presented to that location are detected and discriminated with greater speed and accuracy than stimuli presented to unattended locations. Such attentional orienting processes may occur covertly (i.e. independently of overt behavior such as eye movements), as has been demonstrated in visuospatial attention studies using both central and peripheral cueing (Posner et al., 1978; Posner, 1980; Jonides, 1981; Mü ller and Rabbitt, 1989), and have been interpreted as a consequence of enhanced sensory processing of stimuli at attended locations (Posner, 1980).

However, behavioral studies suggest that there are differences between the effects of central and peripheral cueing of locations. Specifically, such studies suggest that two different attentional mechanisms underlie attention shifts: one voluntary (or endogenous), associated with central cueing, and one involuntary (or exogenous), triggered by peripheral cues (Jonides, 1981; Briand and Klein, 1987; Mü ller and Rabbitt, 1989).

The time course of cueing effects also appears to differ between central and peripheral cues. Peripheral cues trigger a fast automatic shift of attention: the reaction time (RT) facilitation by validly cued targets occurs with stimulus onset asynchronies (SOAs) as short as 50-100 ms (Posner and Cohen, 1984). When a peripheral cue is not informative of the target position, with longer SOAs (300 ms or more), RTs to validly cued targets are slower than RTs to invalidly cued targets (Posner and Cohen, 1984). This effect is known as 'inhibition of return' (IOR). However, when the peripheral cue is informative of the target position, the cue validity effect continues at SOAs of $300 \mathrm{~ms}$ or more (Wright and Richard, 2000). Findings of this type have been interpreted as the result of a voluntary orienting process induced by informative-peripheral cueing with long SOAs, overcoming the IOR effect. Symbolic central cues appear to induce an endogenous, voluntary shift of attention with a relatively slow onset time (at least $200 \mathrm{~ms}$ ) (Mü ller and Rabbitt, 1989), and which is persistent, less dependent on cue-to-target SOA (Yamaguchi et al., 1994), and not prone to IOR effects (Posner and Cohen, 1984).

Covert visuospatial orienting in response to central and peripheral cues has been also investigated in event-related potential (ERP) studies. These studies have revealed that facilitation of RT by central cues is accompanied by enhancements of the P1 and N1 components for stimuli at validly cued locations as compared to stimuli at invalidly cued locations (Mangun et al., 1987; Mangun and Hillyard, 1991). The modulation of the amplitude of the P1 component, starting about 80 ms poststimulus, would represent the earliest effect of spatial attention on visual processing (Hillyard et al., 1999). It has been suggested that this modulation reflects a sensory gain mechanism that results in enhanced perceptual processing of attended stimuli (Mangun and Hillyard, 1990; Mangun et al., 
1993). Recent experiments using ERP source localization techniques (Mangun et al., 1993; Gó mez et al., 1994; Johannes et al., 1995), as well as experiments combining ERP recordings with functional imaging (positron emission tomography and functional magnetic resonance imaging) (Heinze et al., 1994; Mangun et al., 1997; Martínez et al., 1999), have offered evidence that this gating process is accomplished within extrastriate cortical structures (the fusiform gyrus and surrounding areas).

The involuntary attention effects of peripheral cueing on early visual processing are less clear, with different authors having reported different effects on P1. Hillyard et al. (1994) compared the effects of central and peripheral cues on ERPs. In both cases, valid cues elicited an enhancement of the N1 and $\mathrm{N} 2$ components, while valid central cues, but not valid peripheral cues, increased P1.

This failure to detect any effect of peripheral cueing on P1 was surprising, as the predictive value of the cues and the long SOAs used $(600-800 \mathrm{~ms})$ would be expected to elicit voluntary attentional shifts similar to the orienting processes induced by symbolic central cues (Mangun, 1995). Hillyard et al. (1994) concluded that these two types of cue differ in their ability to invoke the earliest type of attentional selection. Eimer (1994) also studied the effects of peripheral cues on ERP using a cue-totarget SOA of $700 \mathrm{~ms}$, and found no P1 enhancement for validly cued stimuli; on the contrary, P1 was larger in invalid than in valid trials. Hopfinger and Mangun (1998) reported similar results using uninformative-peripheral cues with long SOAs. Only Anllo-Vento (1995) has reported validity effects on P1/N1 amplitude with long SOAs.

Since behavioral studies have shown that the optimal effect of peripheral cues is observed with SOAs of about 100 - 175 ms (Müller and Rabbitt, 1989), the negative results obtained by Hillyard et al. (1994) and Eimer (1994) might be attributable to the longer SOAs used in these studies. It is possible that central and peripheral cueing modulate stimulus processing in the visual cortex with different time courses, as is the case with behavioral responses. Nevertheless, results have also been inconsistent in experiments using short SOAs: Lubbe and Woestenburg (1997) detected a contralateral enhancement of the posterior P150 component using peripheral cueing $100-300 \mathrm{~ms}$ prior to the target, and Hopfinger and Mangun (1998) found similar validity effects on P1 using uninformative-peripheral cues with short SOAs. Similar results were also reported recently by $\mathrm{Fu}$ et al. (2001) using informative-peripheral cues. However, Anllo-Vento (1995) did not detect any validity effect on P1/N1 using cues with an SOA of $200 \mathrm{~ms}$.

As explained above, the informative value of peripheral cues is another factor influencing their facilitatory effects and modulating the time course of these effects. To date, only a few studies have investigated the purely automatic effects of uninformative cueing on ERPs. As noted above, Hopfinger and Mangun (1998) observed P1 modulations by uninformative cues that were consistent 
with a facilitatory effect changing to an inhibitory effect when SOA increases; Eimer (1994) and McDonald et al. (1999) also reported smaller P1 to validly cued targets with long SOAs.

The present study was designed to help clarify the effects of peripheral cueing on early visual processing, taking into account these considerations about the influence of cue-to-target interval and the informative value of the cue. To this end, we repeated Hillyard et al. (1994) study, but systematically varied cue-to-target SOA from 100 to $700 \mathrm{~ms}$, with the aim of assessing in detail the time course of the attentional orienting effects of central and peripheral cues on P1 amplitude. Furthermore, we included an additional experimental condition with uninformative-peripheral cueing (i.e. cue validity 50\%), with the aim of obtaining additional evidence about the effects of the automatic orienting elicited by this type of cue.

\section{Methods}

\subsection{Subjects}

Fifteen paid volunteers (12 female, 3 male), aged between 19 and 23 years ( $M=20$ years), took part in the experiment ${ }^{1}$. Informed consent was obtained from each participant. All the subjects were righthanded, had normal or corrected-to-normal vision, and had no history of psychiatric or neurological disorders.

\subsection{Stimuli and apparatus}

The subjects were seated in a comfortable armchair in an electrically isolated, sound- and lightattenuated room, with response buttons under their left and right hands. A computer screen was placed $100 \mathrm{~cm}$ in front of the subject's eyes and carefully positioned so that the stimuli (white-on-black) were presented directly horizontal to the subject's line of vision. A fixed cross was presented continuously at the center of the screen. In the Informative-Central Cue condition, each trial began with an arrow flashed at the fixation point for $34 \mathrm{~ms}$ that pointed either to the left or right visual field on a random basis (each with a probability of 0.50 ). The cue correctly indicated target location in $75 \%$ of trials (i.e. cue validity $75 \%$; valid trials). The target stimuli were either short $\left(1.7^{\circ} \times 0.7^{\circ}\right)$ or long $\left(2.1^{\circ} \times 0.7^{\circ}\right)$ vertical bars flashed for $75 \mathrm{~ms}$ in the left or right visual field at a horizontal distance of 6.48 from the central cross. The target locations were each defined by four continuously present dots that formed the corners of a vertical rectangle $\left(1.5^{\circ} \times 1.1^{\circ}\right)$ centered on the target's position. The interval from cue onset to target onset (SOA) had four values of 100 ,

\footnotetext{
${ }^{1}$ In the uninformative-peripheral cue condition, the data from one subject was discarded because evaluations of her horizontal EOG revealed systematic eye movements to the cued location. 
300, 500 and $700 \mathrm{~ms}$, which varied between blocks. The intertrial intervals were $1800 \mathrm{~ms}$. In the Informative-Peripheral Cue condition, stimuli and procedures were identical to those previously described for the Informative-Central Cue condition, except for the character of the cue: the trials began with a peripheral cue that consisted of a brief displacement of the dots that marked one of the target locations. The four dots were extinguished and replaced for $50 \mathrm{~ms}$ by another four dots that formed a new outline rectangle of $1.1^{\circ} \times 0.5^{\circ}$. The original dots were then restored, giving the appearance that the continuously present marker dots had jumped toward and then away from each other. The cue correctly indicated target location in $75 \%$ of trials (i.e. cue validity $75 \%$; valid trials). The cue-target SOAs were identical to those in the Informative-Central Cue condition. In the Uninformative-Peripheral Cue condition, the stimuli and procedures were identical to those in Informative-Peripheral Cue condition, except that the cue correctly indicated target location in only $50 \%$ of trials (i.e. cue validity $50 \%$; valid trials).

\subsection{Procedure}

For all conditions, subjects maintained eye fixation on the central cross, and were required to discriminate the height of the target bar for each trial, pressing one button with one hand for short bars and another button with the other hand for long bars, as quickly and accurately as possible in each trial. The assignment of the response hand was counterbalanced across subjects. The subjects were informed of the probabilities of the valid and invalid trial types and were told to make use of this information to maximize their performance. In order to familiarize the subjects with these specific task requirements, several training trials were run at the beginning of the experiment. Each experimental condition consisted of 4 trial blocks, according to the SOA variable, resulting in a total of 12 experimental blocks. Each block consisted of 240 trials. The order of the blocks within each experimental condition was counterbalanced for order effects within and across subjects. In the Informative-Central Cue and Informative-Peripheral Cue conditions, 180 (75\%) of the 240 trials per block were valid. In the Uninformative-Peripheral Cue condition, $120(50 \%)$ of the 240 trials were valid. Each condition lasted approximately $35 \mathrm{~min}$. Short breaks were allowed between conditions, with length of the break controlled by the subjects. The order of the conditions was counterbalanced across subjects.

\subsection{Recording}

The electroencephalogram (EEG) was recorded with tin electrodes from T5 and T6 (according to the 10 - 20 system), from PL and PR (located halfway between Pz and the ear canal), and from OL and OR (located halfway between $\mathrm{O} 1$ and $\mathrm{T} 5$, and $\mathrm{O} 2$ and $\mathrm{T} 6$, respectively). All electrodes were referred to an electrode placed over the nose. The horizontal electrooculogram (EOG) was recorded bipolarly from electrodes at the outer canthi of both eyes, and the vertical EOG was recorded from electrodes 
placed supraorbitally and infraorbitally to the left eye. EEG was amplified $10 \mathrm{~K}$ and filtered using a bandpass filter of $0.05-100 \mathrm{~Hz}$. EEG and EOG were sampled with a digitization rate of $500 \mathrm{~Hz}$. Impedances were kept at $10 \mathrm{~K} \Omega$ or less. Reaction times were recorded for each trial.

\subsection{Data analysis}

The EEG and EOG were digitally filtered off-line with a $0.1-30 \mathrm{~Hz}$ bandpass filter, and were epoched into periods of $1000 \mathrm{~ms}$, from $100 \mathrm{~ms}$ prior to the onset of the target to $900 \mathrm{~ms}$ post-target. Trials with eyeblinks, horizontal eye movements, or response errors were excluded from analysis. The EEG was averaged separately for all combinations of task conditions (visual field: right/left; trial validity: valid/ invalid; type of cue: informative-central/informative-peripheral/uninformative-peripheral; cue-totarget SOAs: 100/300/500/700 ms), resulting in 48 average waveforms for each subject and electrode site. All measurements were taken relative to the mean voltage of the pre-target interval. To avoid overlapping of ERPs elicited by cues and targets in the conditions with short SOAs, a correction was applied as follows: The ERPs elicited by cues (left and right hemifield separately) were obtained in the SOA-700 condition (epoch 0-700 ms), which can reasonably be assumed not to overlap with the subsequent target response. These cue ERPs were then time-locked and subtracted from the ERPs elicited by the targets in the SOA-100 and SOA-300 conditions, so eliminating the putative effects of the electrophysiological response to the cue on the ERPs elicited by the targets. (See the appendix for a further description of the procedure to correct for possible ERP overlapping).

The averaged ERPs were analyzed with a semi-automatic peak detection program, which examined a latency window of 75-165 ms for peak P1. Peaks were then verified and adjusted by visual inspection. Amplitude values were automatically exported to an ASCII file for subsequent analysis.

Repeated-measures analyses of variance (ANOVA) were performed on amplitude values with the within-subject factors electrode (6 levels), type of cue (3 levels), validity (2 levels), laterality (target stimuli in the contralateral vs. ipsilateral visual field relative to the electrode location, 2 levels), and cue-to-target SOA (4 levels). Significance levels were determined using degrees of freedom after applying the Greenhouse-Geisser correction when the data violated the assumption of sphericity. Posthoc comparisons were performed using the Bonferroni adjustment for multiple comparisons. For the reaction time data, repeated-measure ANOVAs were carried out for the factors type of cue, cue-totarget SOA, validity, and visual field of stimulus. 


\section{Results}

\subsection{Behavioral performance}

Mean RT as a function of SOA, visual field and validity for informative-central, informativeperipheral and uninformative-peripheral cueing conditions are presented in Table 1 . There was only a significant main effect of validity $(\mathrm{F}(1,14)=82.461, \mathrm{P}=0.0005)$, showing that reaction times were significantly faster for validly cued targets than for invalidly cued targets. The magnitude of the validinvalid difference in RT varied significantly among cueing conditions, as indicated by the significant type of cue $\mathrm{x}$ validity interaction $(\mathrm{F}(2,28)=7.405, \mathrm{P}=0.003)$. Pairwise analyses showed that the validity effect was significantly larger for informative-central cues (563 \pm 55 vs. $598 \pm 58)$ than for uninformative-peripheral cues $(569 \pm 71$ vs. $584 \pm 69)(\mathrm{F}(1,14)=15: .47, \mathrm{P}=0.002)$ and for informative-peripheral cues $(558 \pm 66$ vs. $583 \pm 62)$ than for uninformative-peripheral cues $(\mathrm{F}(1,14)=$ 5.700, $\mathrm{P}=0.032$ ).

\section{Table 1}

Significant validity x $\operatorname{SOA}(\mathrm{F}(3,42)=6.695, \mathrm{P}=0.001)$ and type of cue $x \operatorname{SOA} \times$ validity $(\mathrm{F}(6,84)=$ $4.845, \mathrm{P}=0.0005)$ interactions were also detected. Separate analyses for each cueing condition revealed that the validity $x$ SOA interaction persisted for informative-peripheral cues $(\mathrm{F}(3,42)=$ 5.766, $\mathrm{P}=0.002)$ and for uninformative-peripheral cues $(\mathrm{F}(3,42)=14.486, \mathrm{P}=0.0005)$. For informative-peripheral cues, the validity effect was significant at SOAs of $100 \mathrm{~ms}(\mathrm{~F}(1,14)=43.537$, $\mathrm{P}=0.0005), 300 \mathrm{~ms}(\mathrm{~F}(1,14)=24.360, \mathrm{P}=0.0005)$ and $500 \mathrm{~ms}(\mathrm{~F}(1,14)=11.663, \mathrm{P}=0.004)$. For uninformative-peripheral cues, the largest validity effect was found at $100 \mathrm{~ms} \operatorname{SOA}(\mathrm{F}(1,14)=$ $100.084, \mathrm{P}=0.0005$ ), while there was no significant effect at 300,500 or $700 \mathrm{~ms}$ SOAs.

There was no significant validity x SOA interaction for informative-central cues: validity effects on RT were significant at all SOAs.

Although no statistically significant main effects were found for the factors SOA and type of cue, there was a significant interaction between them $(F(6,84)=2.976, P=0.011)$, as a result of significantly slower RTs after informative-central cues than after informative-peripheral cues $(F(1,14)$ $=6.077, \mathrm{P}=0.027)$ or uninformative-peripheral cues $(\mathrm{F}(1,14)=5.408, \mathrm{P}=0.036)$ at $100 \mathrm{~ms}$ SOA.

The factor visual field had no significant effects, nor did it show significant interactions with other variables. 


\subsection{Event-related potentials}

Grand-averaged ERPs (15 subjects; valid and invalid targets; occipital, parietal, and temporal electrodes; ipsilateral and contralateral to the target location) are shown in Figs. 1-3 for informativeperipheral, uninformative-peripheral and informative-central cueing, respectively. The P1 component was largest at occipital locations (OL and $\mathrm{OR}$ ) in all conditions, as indicated by a significant effect of electrode $(\mathrm{F}(5,70)=15.730, \mathrm{P}=0.0005, \varepsilon=0.612)$. The SOA main effect was significant $(\mathrm{F}(3,42)=$ 9.642, $\mathrm{P}=0.0005)$ : specifically, the largest $\mathrm{P} 1$ amplitudes were found at 300 and $700 \mathrm{~ms}$ SOA, differing significantly from those at 500 and $100 \mathrm{~ms}$ SOA (300 vs. $100: \mathrm{P}=0.022 ; 300$ vs. $500: \mathrm{P}=$ $0.037 ; 700$ vs. $100: \mathrm{P}=0.013 ; 700$ vs. 500 : $\mathrm{P}=0.009$ ).

Figures 1, 2, 3

There was not a significant main effect of type of cue, but this factor significantly interacted with SOA $(F(6,84)=4.338, P=0.008, \varepsilon=0.539)$, due to $\mathrm{P} 1$ amplitude being significantly smaller for informative-central cues than for informative- $(\mathrm{P}=0.004)$ and uninformative- $(\mathrm{P}=0.002)$ peripheral cues at $500 \mathrm{~ms}$ SOA. There were no significant effects of laterality, and no significant interactions between this factor and other factors.

Although no main effect of Validity was detected, there was a significant validity x SOA interaction $(\mathrm{F}(3,42)=4.056, \mathrm{P}=0.032, \varepsilon=0.613)$. There were also significant 3-way interactions between electrode, validity and SOA $(\mathrm{F}(15,210)=2.288, \mathrm{P}=0.049, \varepsilon=0.364)$ and electrode, type of cue and validity $(\mathrm{F}(10,140)=4.491, \mathrm{P}=0.007, \varepsilon=0.317)$. Therefore, to evaluate in detail the time course of the validity effect on P1 amplitude, separate analyses were carried out for each electrode pair in the parietal (PL/PR), temporal (T5/T6) and occipital (OL/OR) scalp locations. These ANOVAs included an additional variable, namely hemisphere of recording (left/right). Considering the three task conditions together, the validity $x$ SOA interaction was significant for the occipital $(F(3,42)=4.997, \mathrm{P}$ $=0.020, \varepsilon=0.565)$ and parietal $(\mathrm{F}(3,42)=4.544, \mathrm{P}=0.020, \varepsilon=0.664)$ locations. Thus, additional ANOVAs were carried out for each cueing condition only at these locations. The main results of these analyses are synthesized in Table 2. Only the effects of validity and its interactions with the other variables included in the analysis are reported, since these are the most relevant to the goals of this study.

\subsubsection{Informative-peripheral cueing}

There was a significant validity x SOA interaction for occipital scalp locations $(\mathrm{F}(3,42)=4.408, \mathrm{P}=$ 0.009), but not for parietal locations (see Table 2). Separate analyses of the validity effect for each SOA at occipital locations (see Table 3), revealed that the P1 amplitude was larger for valid than for 
invalid trials at $100 \mathrm{~ms} \mathrm{SOA}(\mathrm{F}(1,14)=6.253, \mathrm{P}=0.025)$, but smaller for valid than for invalid trials at $500 \mathrm{~ms} \mathrm{SOA}(\mathrm{F}(1,14)=5.868, \mathrm{P}=0.030)$.

Tables 2, 3

\subsubsection{Uninformative-peripheral cueing}

As shown in the Table 2, there was a significant effect of validity $(\mathrm{F}(1,14)=6.841, \mathrm{P}=0.020)$ at parietal sites, indicating that P1 amplitude was larger for invalid than for valid trials. The effect was more prominent in the left hemisphere, as revealed by the significant hemisphere of recording $\mathrm{x}$ validity interaction $(\mathrm{F}(1,14)=4.784, \mathrm{P}=0.046)$. In addition, there was a significant validity $\mathrm{x}$ SOA interaction at parietal $(\mathrm{F}(3,42)=6.536, \mathrm{P}=0.001)$ and occipital $(\mathrm{F}(3,42)=6.395, \mathrm{P}=0.001)$ locations. Additional ANOVAs for each SOA showed that at parietal sites (see Table 3), P1 was larger for invalid than for valid trials at $300 \mathrm{~ms} \operatorname{SOA}(\mathrm{F}(1,14)=13.021, \mathrm{P}=0.003), 500 \mathrm{~ms} \mathrm{SOA}$ $(\mathrm{F}(1,14)=4.793, \mathrm{P}=0.046)$ and $700 \mathrm{~ms} \operatorname{SOA}(\mathrm{F}(1,14)=7.830, \mathrm{P}=0.014)$. The effect on $\mathrm{P} 1$ amplitude with $300 \mathrm{~ms}$ SOA was greater at the left electrode, as revealed by the significant hemisphere of recording $\mathrm{x}$ validity interaction $(\mathrm{F}(1,14)=5.147, \mathrm{P}=0.040)$. At occipital locations, the $\mathrm{P} 1$ amplitude was larger for valid than for invalid trials at $100 \mathrm{~ms} \mathrm{SOA}(\mathrm{F}(1,14)=6.884, \mathrm{P}=0.020)$; by contrast, at $700 \mathrm{~ms}$ SOA, P1 amplitude was smaller for valid than for invalid trials $(\mathrm{F}(1,14)=$ 7.936, $\mathrm{P}=0.014)($ see Table 3$)$.

\subsubsection{Informative-central cueing}

There were no significant differences between valid and invalid trials, and none of the variables considered showed significant interactions with validity.

\section{Discussion}

\subsection{Behavioral performance}

As in previous RT studies, the subjects in the present experiment were significantly faster at responding to targets in validly cued locations than in invalidly cued locations. These validity effects on RT differed among cueing conditions. A significantly larger validity effect was observed for informative-central and informative-peripheral cues than for uninformative-peripheral cues. These differences indicate that spatial cues were more effective in orienting attention to the cued location when they provided information about the probable location of the target. 
These validity effects revealed differences as a function of SOA in both peripheral cueing conditions. Peripheral cues led to RT facilitation with the shortest SOA (100 ms), and this facilitation was not affected by cue informativeness (i.e. probability of valid cue 75 or 50\%). RT facilitation of this type has been attributed to automatic attraction of attention by the cue (Jonides, 1981). With SOAs longer than $100 \mathrm{~ms}$, different patterns were observed, depending on cue informativeness. With informativeperipheral cueing, a validity effect was observed at 300 and $500 \mathrm{~ms} \mathrm{SOA}$, but not at $700 \mathrm{~ms}$ SOA. These findings are consistent with the behavioral results obtained in previous ERP studies, that have investigated peripheral cueing effects with several SOAs. Anllo-Vento (1995) reported faster RTs for validly than invalidly cued targets when the SOA was short (200 ms) than when it was longer (600 ms). Yamaguchi et al. (1994) similarly found that responses were faster for validly than for invalidly cued targets at an SOA of $200 \mathrm{~ms}$, but not with longer SOAs (500 and $800 \mathrm{~ms}$ ). These results also agree with behavioral studies showing that when a peripheral cue provides information about the probable target location, the validity effect observed with short SOAs continues to be present with SOAs of $300 \mathrm{~ms}$ or more (Wright and Richard, 2000), although it declines in magnitude as SOA increases (Mü ller and Rabbitt, 1989). These effects have been interpreted as the result of a voluntary orienting process elicited by highly predictive peripheral cueing at long SOAs.

With uninformative-peripheral cues, by contrast, the validity effect was only present at the shortest SOA, and disappeared at the longer SOAs. The results obtained in this condition are consistent with the findings of Hopfinger and Mangun (1998), who reported faster RTs to targets at the cued location than to targets at the non-cued location with short SOAs (34-234 ms) but not with long SOAs (566$766 \mathrm{~ms}$ ). Eimer (1994) also detected no validity effects on RT with uninformative-peripheral cues at an SOA of $700 \mathrm{~ms}$. These results suggest that the RT facilitation effect of cues with SOA of $300 \mathrm{~ms}$ is due to voluntary orienting processes, present with informative cues but absent with uninformative cues.

The absence of any validity effect on RTs at long SOAs would suggest that inhibition of return was not elicited by the uninformative-peripheral cues. The IOR effect consists of slower RTs to cued locations as compared with uncued locations (Posner and Cohen, 1984). This absence may be attributable to the use of a discrimination task. Previous research (Terry et al., 1994) has suggested that such tasks reduce the probability of obtaining RT inhibition. However, other authors have reported evidence against this hypothesis (e.g. Lupiáñez et al., 1997; Handy et al., 1999), suggesting that IOR may occur in discrimination tasks. Another possible explanation is that the absence of an IOR effect is due to the fact that only a single location was cued in the present experiment. In the typical paradigm designed to investigate the effects of IOR (Posner and Cohen, 1984), the peripheral cue is followed by a second (central) cue presented at fixation, before the target is presented. Posner 
and Cohen (1984) reported that IOR does not appear to be elicited by peripheral cueing if attention is not drawn away from the cued location back to the neutral fixation point.

In contrast to the effects of peripheral cueing, the RT validity effect of central cues did not depend on SOA; responses were faster for valid than for invalid cues at all SOAs, indicating that central cues produce a more sustained facilitation of performance, a result that is in agreement with previous studies (Mü ller and Rabbitt, 1989; Yamaguchi et al., 1994).

\subsection{Event-related potentials}

One of the main objectives of this study was to investigate the time course of peripheral cueing effects on early visual processing. The results obtained with peripheral cueing at occipital sites revealed that, with a SOA of $100 \mathrm{~ms}$, the amplitude of P1 was larger when the target was validly cued than when it was invalidly cued. However, P1 amplitude was larger for invalid than for valid trials with SOA 500 ms (informative-peripheral cues) or $700 \mathrm{~ms}$ (uninformative-peripheral cues); for the latter type of cue, this effect extended to SOAs of 300 and $500 \mathrm{~ms}$ at parietal sites.

The enhancement of the P1 component to valid targets with informative-peripheral cueing after a short SOA (100 ms) supports the hypothesis that the absence of a validity effect in Hillyard et al. (1994) study could be due to the use of overly long SOAs (600-800 ms). This result agrees with previous findings (Lubbe and Woestenburg, 1997; Fu et al., 2001). On the other hand, the P1 validity effect observed with uninformative-peripheral cueing with SOA of $100 \mathrm{~ms}$ is consistent with the findings of Hopfinger and Mangun (1998). Furthermore, these ERP results are consistent with the facilitation of RT in valid as opposed to invalid trials with an SOA of $100 \mathrm{~ms}$.

The effect of cue validity on P1 amplitude, which has been previously observed in several central cueing ERP studies (Mangun et al., 1987; Mangun and Hillyard, 1991), has been interpreted in terms of a sensory gain control mechanism in extrastriate cortical structures, resulting in enhanced perceptual processing of validly cued stimuli (Mangun and Hillyard, 1990). Within this model, it might be suggested that these ERP data provide evidence that peripheral cueing leads to facilitation of the perceptual processing of stimuli presented in cued locations when the SOA is short enough. This sensory gain may be responsible for the RT benefits in behavioral execution. These findings suggest that automatic attraction of attention by a peripheral cue results in improved sensory processing at the cued location for a short period after presentation of the cue.

In contrast, at the longest SOAs, P1 amplitude was larger for invalidly cued targets than for validly cued targets with SOAs of 300, 500 and $700 \mathrm{~ms}$ for uninformative-peripheral cueing and with SOA of $500 \mathrm{~ms}$ for informative-peripheral cueing. 
The reduction of P1 amplitude for valid trials with long SOA with uninformative-peripheral cueing is consistent with previous findings (Eimer, 1994; Hopfinger and Mangun, 1998; McDonald et al., 1999). This may reflect an IOR effect (Posner and Cohen, 1984). However, this possibility seems unlikely because of the absence of any validity effects on RT at long SOAs. Reduced P1 to valid trials with peripheral cues and long SOAs in the absence of any behavioral IOR has also been found in previous studies: Eimer (1994) reported a similar reduction with SOA of $700 \mathrm{~ms}$, and Hopfinger and Mangun (1998) with SOAs of 566-766 ms. In the present study, the systematic variation of SOA, from 100 to $700 \mathrm{~ms}$ in steps of $200 \mathrm{~ms}$, allowed us to observe that this effect is already present with an SOA of $300 \mathrm{~ms}$.

With informative-peripheral cueing, P1 was larger for invalid trials than for valid trials only with an SOA of $500 \mathrm{~ms}$. However, the behavioral results showed a facilitation of performance with SOAs of 300 and 500 ms. Hillyard et al. (1994) found no significant effect on P1 at long SOAs, accompanied by facilitation of RT. Mangun (1995) suggested that this ERP result might be related to the IOR effect, even though a behavioral IOR effect was not observed. This author proposed that the absence of an IOR effect on RT with informative-peripheral cueing may be due to two competing influences with opposing effects: a voluntary orienting of attention that leads to faster responses to validly cued targets, and an IOR effect that slows responses to validly cued targets. Thus, the absence of the P1 attention effect may reflect an IOR effect, whereas the voluntary orienting might be associated with amplitude enhancements in later ERP components (N1, N2 and P3), as well as faster responses to validly cued targets.

The findings of the present study with informative-peripheral cueing might similarly be interpreted as the result of the confluence of two opposing effects: a voluntary orienting process elicited by the informative value of the cue, starting at SOA $300 \mathrm{~ms}$ as suggested by the behavioral results, and an inhibitory effect. The voluntary orienting process might compensate the inhibitory effects of peripheral cues at cued locations with long SOAs. The uninformative-peripheral cue results are consistent with this hypothesis: with this type of cue, voluntary orienting does not occur, and the inhibitory effect at the cued location would be maintained at all longest SOAs. The significant enhancement of P1 amplitude for invalid trials at $500 \mathrm{~ms}$ SOA with informative-peripheral cueing might reflect the time after cue presentation during which the inhibitory effect is highest, overcoming the opposing effect of the voluntary orienting process. The facilitation of behavioral performance with informative-peripheral cueing with 300 and $500 \mathrm{~ms}$ SOAs suggests that this facilitation is produced at a later processing stage. What this stage is must be determined by analysis of the later ERP components.

However, these results disagree with the P1/N1 enhancement for valid trials reported by Anllo-Vento (1995) in a study in which SOAs of $600 \mathrm{~ms}$ were used. These differences may be attributable to 
differences in task demands between the two studies. Anllo-Vento used a simple RT task, whereas in the present study a choice RT task was used. Some authors (Rafal and Henik, 1994) have provided evidence of differences between detection and discrimination tasks with regard to the time course and the magnitude of the central and peripheral cue effects. Although this interpretation is plausible, further experiments comparing peripheral cueing effects on ERPs in detection and discrimination tasks are needed to assess this hypothesis. It should also be noted that Anllo-Vento measured P1/N1 amplitude; it is likely that their amplitude enhancement was due to N1, which appears as consistently affected by valid cues in several studies (Eimer, 1994; Hillyard et al., 1994).

Another aim of the present study was to investigate P1 amplitude effects of central cueing at several SOAs, in order to examine differences in the time course of facilitatory effects on the visual processing induced by central and peripheral cues. The results obtained in the informative-central cue condition did not show significant differences in P1 amplitude in response to validly and invalidly cued targets, nor any difference in the P1 validity effect depending on SOA, although the behavioral results showed that central cues lead to facilitation of performance.

To date, no studies have been published which compare ERPs recorded in response to target stimuli following central cues at different SOAs, which makes it difficult to evaluate the consistency of the findings obtained in the present experiment. Nevertheless, the absence of significant validity effects on P1 amplitude for central cues with a SOA of $700 \mathrm{~ms}$ differs from the findings of Hillyard et al. (1994), who detected larger P1 amplitudes to valid rather than invalid trials. This discrepancy is surprising, since the central cue condition used in this study is a replication of the experiment of Hillyard et al., except that several additional cue-target SOAs were added. The absence of a validity effect on P1 suggests that voluntary orienting of attention in response to a central cue did not affect visual processing at the P1 level in the present study. Analyses of the later ERP components are necessary to identify psychophysiological indices of the attentional facilitation observed in the behavioral results. Furthermore, such analyses will allow comparison of the effects of the voluntary attention elicited by central and informative-peripheral cueing at later stages of processing.

In summary, the findings obtained in the present study suggest that peripheral cueing facilitates the perceptual processing of stimuli presented at cued locations, even when cues were not predictive of the location of the target, when the SOA was sufficiently short $(100 \mathrm{~ms})$. These results suggest that the automatic attraction of attention by a peripheral cue results in enhanced sensory processing at the cued location. At the longest SOAs, this facilitation did not persist; on the contrary, P1 amplitude was reduced for validly cued targets, suggesting an inhibition of sensory processing at the cued location. The finding that uninformative-peripheral cues led to significantly smaller amplitudes in valid trials at all the longest SOAs, whereas informative-peripheral cues only showed this effect with an SOA of $500 \mathrm{~ms}$, suggests differences in the influence of peripheral cues on early visual processing as a 
function of cue informativeness. This difference might be due to different orienting processes elicited by informative- and uninformative-peripheral cues at long SOAs, as suggested by the behavioral results. Specifically, informative-peripheral cues might elicit a voluntary orienting process, starting at SOA $300 \mathrm{~ms}$, which might compensate the inhibitory effects of peripheral cues at long SOAs. With uninformative-peripheral cues this voluntary orienting would not be present, resulting in a more sustained inhibitory effect at cued locations at all the longest SOAs. The absence of validity effects on P1 for central cues means that it is not possible to confirm that voluntary orienting in response to a central cue affects the stage of visual processing reflected by P1.

\section{Acknowledgements}

This study was supported by Spain's Ministerio de Ciencia y Tecnología (grant BSO2000-0041) and by the Galician Secretaría Xeral de I+D (PGIDT00PSI211102PR, PGIDT01PXI21101PN). The first author holds a postgraduate scholarship from the Spanish Ministerio de Educación, Cultura y Deporte (grant AP99). We thank to Neurobehavioral Systems, Inc. for the free software Presentation, used to design and administer the stimulation paradigm.

\section{Appendix A}

\section{Procedure to correct for possible ERP overlapping}

To avoid overlapping of the ERPs elicited by cues and targets in the shortest SOA conditions, a correction was applied as follows.

Let VR(cue,tar,soa,loc;t) be the averaged ERP recorded at electrode location loc for each cue, target and SOA combination (six values for cue: central right-left, peripheral informative right-left, peripheral non-informative right-left; 4 for target: valid right-left, invalid right-left; four for SOA: $100,300,500,700 \mathrm{~ms}$ ); the variable ' $\mathrm{t}$ ' denotes the time (these functions are assumed throughout this paper to be discrete functions of time). Let us also write $\mathrm{VC}(\mathrm{cue}$, loc; t) for the averaged ERP triggered by a given cue at the electrode location loc, and VT(cue, tar, soa, loc; t) for the averaged ERP elicited by a given target after the corresponding cue. Assuming, as in the Adjar technique (Woldorff, 1993), that both VC and VT are time-invariant and that the electric fields of overlapping ERPs add linearly, the averaged recorded ERP (time-locked to the target), VR, is given by 
VR(cue; tar; soa; loc; t $)$ = VT(cue; tar; soa; loc; t $)+$ VC $($ cue; loc; $t$ + soa $)$

Writing Eq. (1) we implicitly assume, apart from system linearity and time-invariance, that:

- only first-order overlapping effects from the immediately previous cue are taken into account (i.e. no overlapping effects from more remote previous nor immediate subsequent cues and targets are of interest, which is a reasonable assumption since the intertrial intervals were as long as 1800 $\mathrm{ms})$; and

- the shape of the cue ERP (VC) is independent of the SOA (an assumption supported by the fact that the recorded cue response is noticeably equal for all SOAs during the time slot of the corresponding SOA).

Filtering the VR waveform to obtain the desired VT requires having a good estimate of the overlapping response VC. In ERP experiments with only short SOAs and short intertrial intervals, interstimulus jittering is essential in order to enable the application of procedures for estimating $\mathrm{VC}$ like those used in the Adjar level 1 technique. Indeed, the combination of jittering and grandaveraging is what allows us to obtain in these cases a reasonable estimate of the overlapping previous responses and carry out the overlap removal once the previous event distributions are known. However, in our experiment we have recorded VC waveforms for each cue condition (including the cue hemifield) with a long SOA (700 ms) which can reasonably be assumed not to overlap with the subsequent target response. According to (b), these averaged waveforms [denoted henceforth by $\mathrm{VC}_{700}(\mathrm{cue}, \mathrm{loc} ; \mathrm{t})$ ] give us a reasonably good estimate of the VC ERPs for the $700 \mathrm{~ms}$ following the corresponding cue, whatever the SOA may be. We have separately computed the $\mathrm{VC}_{700}$ for each cue condition at each relevant electrode location. Then, for each (cue, tar, soa, loc) condition, filtering can be done by subtracting

VT $($ cue; tar; soa; loc; $\mathrm{t})=\mathrm{VR}(\mathrm{cue} ;$ tar; soa; loc; $\mathrm{t})-\mathrm{VC}_{700}(\mathrm{cue} ;$ loc; $\mathrm{t}+$ soa $)$

The range of validity of Eq. (2) extends to an elapsed time of [700 ms-SOA] since the stimulus onset. Eq. (2) is a particular case of overlap removal when the previous-event normalized distribution approaches a Kronecker-delta in the discrete time domain and the $\mathrm{VC}$ waveforms are known. In our case the absence of jitter for each nominal SOA does not allow the low-pass filtering of the VC contribution to the recorded ERP, so there is no gain in implementing recursive procedures such as Adjar level-2. Furthermore, using such a recursive procedure to estimate the VC contribution does not seem necessary, since the required data can be obtained directly from the $\mathrm{VC}_{700}$ records. 


\section{References}

Anllo-Vento L. Shifting attention in visual space: the effects of peripheral cueing on brain cortical potentials. Int J Neurosci 1995;80:353-70.

Briand KA, Klein RM. Is Posner's 'beam' the same as Treisman's 'glue'? On the relation between visual orienting and feature integration theory. J Exp Psychol Hum Percept Perform 1987;13:228-41.

Eimer M. An ERP study on visual spatial priming with peripheral onsets. Psychophysiology 1994;31:154-63.

Fu S, Fan S, Chen L, Zhuo Y. The attentional effects of peripheral cueing as revealed by two eventrelated potential studies. Clin Neurophysiol 2001;112:172-85.

Gómez CM, Clark VP, Fan S, Luck SJ, Hillyard SA. Sources of attention-sensitive visual eventrelated potentials. Brain Topogr 1994;7:41-51. Handy TC, Jha AP, Mangun GR. Promoting novelty in vision: inhibition of return modulates perceptual-level processing. Psychol Sci 1999;10:157-61.

Heinze HJ, Mangun GR, Burchert W, Hinrichs H, Scholz M, Mü nte TF, Gös A, Scherg M, Johannes S, Hundeshagen H, Gazzaniga MS, Hillyard SA. Combined spatial and temporal imaging of brain activity during visual selective attention in humans. Nature 1994;372:543-6.

Hillyard SA, Luck SJ, Mangun GR. The cuing of attention to visual field locations: analysis with ERP recordings. In: Heinze HJ, Münte TF, Mangun GR, editors. Cognitive electrophysiology. Boston: Birkhäuser;1994. p. 1-25.

Hillyard SA, Vogel EK, Luck SJ. Sensory gain control (amplification) as a mechanism of selective attention: electrophysiological and neuroimaging evidence. In: Humphreys GW, Duncan J, Treisman A, editors. Attention, space and action. Studies in Cognitive Neuroscience. New York: Oxford University Press; 1999. p. 31-53.

Hopfinger JB, Mangun GR. Reflexive attention modulates processing of visual stimuli in human extrastriate cortex. Psychol Sci 1998;9:441-7. Johannes S, Münte TF, Heinze HJ, Mangun GR. Luminance and spatial attention effects on early visual processing. Cogn Brain Res 1995;2:189-205. 
Jonides J. Voluntary versus automatic control over the mind's eye's movement. In: Long JB, Baddeley AD, editors. Attention and performance IX. Hillsdale, NJ: Lawrence Erlbaum Associates; 1981. p. 187-203.

Lubbe RHJ, Woestenburg JC. Modulation of early ERP components with peripheral precues: a trend analysis. Biol Psychol 1997;45:143-58.

Lupiáñez J, Milán EG, Tornay F, Madrid E, Tudela P. Does IOR occur in discrimination tasks? Yes, it does, but later. Percept Psychophys 1997;59:1241-54.

Mangun GR. Neural mechanisms of visual selective attention. Psychophysiology 1995;32:4-18.

Mangun GR, Hillyard SA. Electrophysiological studies of visual selective attention in humans. In: Scheibel AB, Wechsler A, editors. The neurobiological foundations of higher cognitive function. New York: Guilford Press; 1990. p. 271-95.

Mangun GR, Hillyard SA. Modulations of sensory-evoked brain potentials indicate changes in perceptual processing during visual-spatial priming. J Exp Psychol Hum Percept Perform 1991;17:1057-74.

Mangun GR, Hansen JC, Hillyard SA. The spatial orienting of attention: sensory facilitation or response bias? In: Johnson R, Rohrbaugh JW, Parasuraman R, editors. Current trends in event-related potential research. Amsterdam: Elsevier; 1987. p. 118-24.

Mangun GR, Hillyard SA, Luck SJ. Electrocortical substrates of visual selective attention. In: Meyer D, Kornblum S, editors. Attention and performance XIV. Cambridge, MA: The MIT Press; 1993. p. 219-43.

Mangun GR, Hopfinger JB, Kussmaul CL, Fletcher EM, Heinze HJ. Covariations in ERP and PET measures of spatial selective attention in human extrastriate visual cortex. Hum Brain Mapp 1997;5:273-9.

Martínez A, Anllo-Vento L, Sereno MI, Frank LR, Buxton RB, Dubowitz DJ, Wong EC, Hinrichs H, Heinze HJ, Hillyard SA. Involvement of striate and extrastriate visual cortical areas in spatial attention. Nat Neurosci 1999;2:364-9.

McDonald JJ, Ward LM, Kiehl KA. An event-related brain potential study of inhibition of return. Percept Psychophys 1999;61:1411-23. 
Müller HJ, Rabbitt PMA. Reflexive and voluntary orienting of visual attention: time course of activation and resistance to interruption. J Exp Psychol Hum Percept Perform 1989;15:31530.

Posner MI. Orienting of attention. Q J Exp Psychol 1980;32:3-25.

Posner MI, Cohen Y. Components of visual orienting. In: Bouma H, Bouwhuis DG, editors. Attention and performance X: control of language processes. Hillsdale, NJ: Lawrence Erlbaum Associates;1984. p. 531-56.

Posner MI, Nissen MK, Ogden WC. Attended and unattended processing modes: the role of set for spatial location. In: Pick HL, Saltzman E, editors. Modes of perceiving and processing information. Hillsdale, NJ: Lawrence Erlbaum Associates; 1978. p. 137-57.

Rafal R, Henik A. The neurology of inhibition. Integrating controlled and automatic processes. In: Dagenbach D, Carr TH, editors. Inhibitory processes in attention, memory and language. San Diego, CA: Academic Press; 1994. p. 1-51.

Terry KM, Valdes LA, Neill WT. Does 'inhibition of return' occur in discrimination tasks? Percept Psychophys 1994;55:279-86.

Woldorff MG. Distortion of ERP averages due to overlap from temporally adjacent ERPs: analysis and correction. Psychophysiology 1993;30:98-119.

Wright RD, Richard CM. Location cue validity affects inhibition of return of visual processing. Vision Res 2000;40:2351-8.

Yamaguchi S, Tsuchiya H, Kobayashi S. Electroencephalographic activity associated with shifts of visuospatial attention. Brain 1994;117:553-62. 
Tables

Table 1. Mean RT (ms) as a function of SOA, visual field and cue validity for informative-central, informative-peripheral and uninformative-peripheral cueing conditions ${ }^{\mathrm{a}}$

\begin{tabular}{l}
\hline \multicolumn{3}{c}{ SOA 100 ms } & \multicolumn{2}{c}{ SOA 300 $\mathbf{~ m s}$} & \multicolumn{2}{c}{ SOA 500 ms } & \multicolumn{2}{c}{ SOA 700 ms } \\
\hline
\end{tabular}

Table 2. Summary of statistical analyses (P values) of the effect of validity on P1 amplitude at occipital and parietal locations in the informative-central, informativeperipheral and uninformative-peripheral cueing conditions

\begin{tabular}{llccc}
\hline \multicolumn{1}{c}{ Type of cue } & & Validity & Validity x SOA & Validity $\mathbf{x}$ \\
\hline Informative & OL/OR & $\mathrm{ns}$ & 0.009 & $\mathrm{~ns}$ \\
Peripheral & PL/PR & $\mathrm{ns}$ & $\mathrm{ns}$ & $\mathrm{ns}$ \\
Uninformative & OL/OR & $\mathrm{ns}$ & 0.001 & $\mathrm{~ns}$ \\
Peripheral & PL/PR & 0.020 & 0.001 & 0.046 \\
Informative & OL/OR & $\mathrm{ns}$ & $\mathrm{ns}$ & $\mathrm{ns}$ \\
Central & PL/PR & $\mathrm{ns}$ & $\mathrm{ns}$ & $\mathrm{ns}$ \\
\hline
\end{tabular}

ns $=$ not significant

\section{Post-print (final dråft post-refereeing)}


Table 3. Summary of statistical analyses (P values) of the effect of validity on P1 amplitude at occipital and parietal locations for each SOA in the informative-peripheral and uninformative-peripheral cueing conditions

\begin{tabular}{llcc}
\hline & & $\begin{array}{c}\text { Informative } \\
\text { peripheral }\end{array}$ & $\begin{array}{c}\text { Uninformative } \\
\text { peripheral }\end{array}$ \\
\hline SOA 100 & OL/OR & 0.025 & 0.020 \\
& PL/PR & $\mathrm{ns}$ & $\mathrm{ns}$ \\
SOA 300 & OL/OR & $\mathrm{ns}$ & $\mathrm{ns}$ \\
& PL/PR & $\mathrm{ns}$ & 0.003 \\
SOA 500 & OL/OR & 0.030 & $\mathrm{~ns}$ \\
& PL/PR & $\mathrm{ns}$ & 0.046 \\
SOA 700 & OL/OR & $\mathrm{ns}$ & 0.014 \\
& PL/PR & $\mathrm{ns}$ & 0.014 \\
\hline
\end{tabular}

$\mathrm{ns}=$ not significant 


\section{Figures}

\section{INFORMATIVE-PERIPHERAL CUEING}

SOA 100

CONTRALATERAL

IPSILATERAL

SOA 300
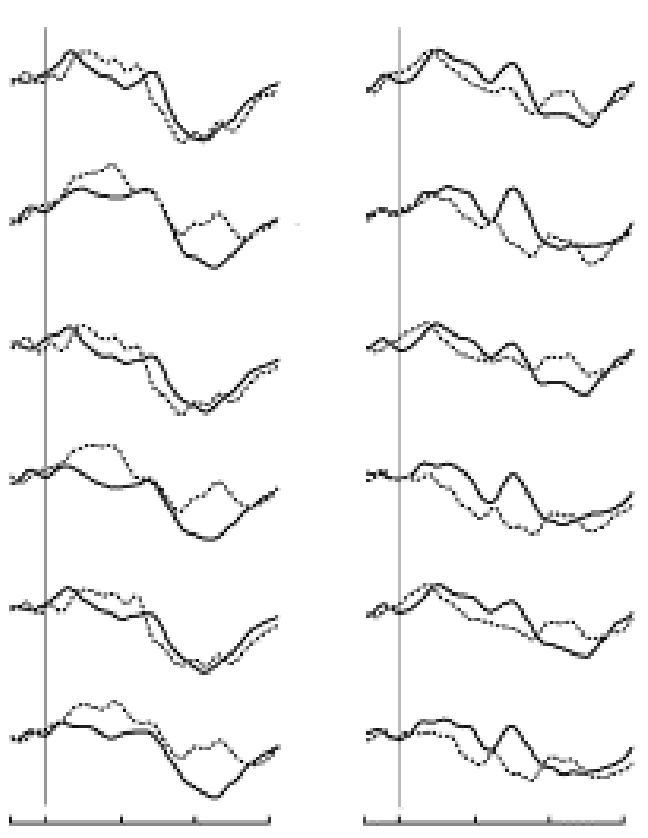

ol

CONTRALATERAL

IPSILATERAL

op

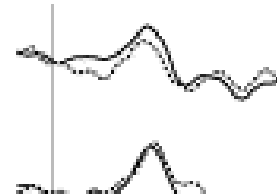

pt
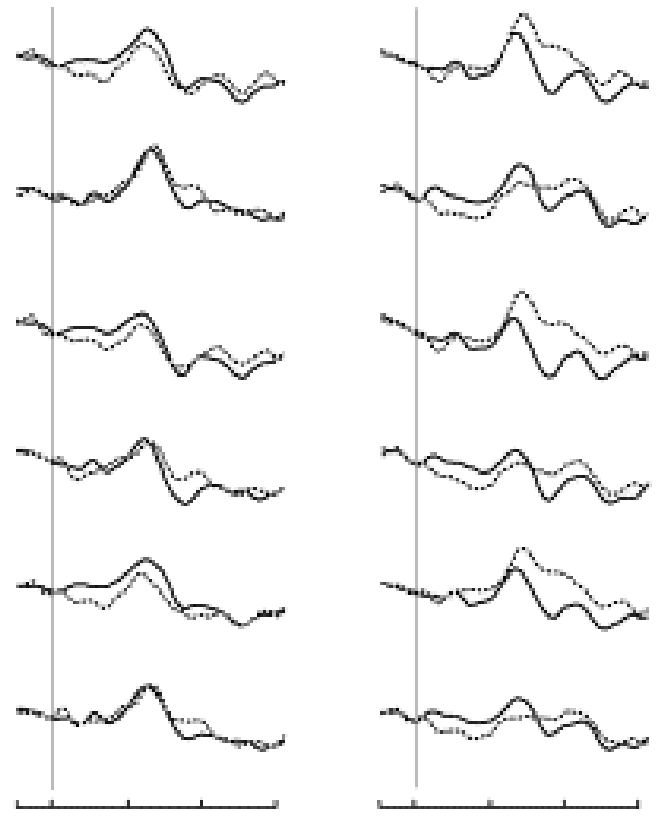

SOA 500

CONTRALATEAAL

IPSILATERAL
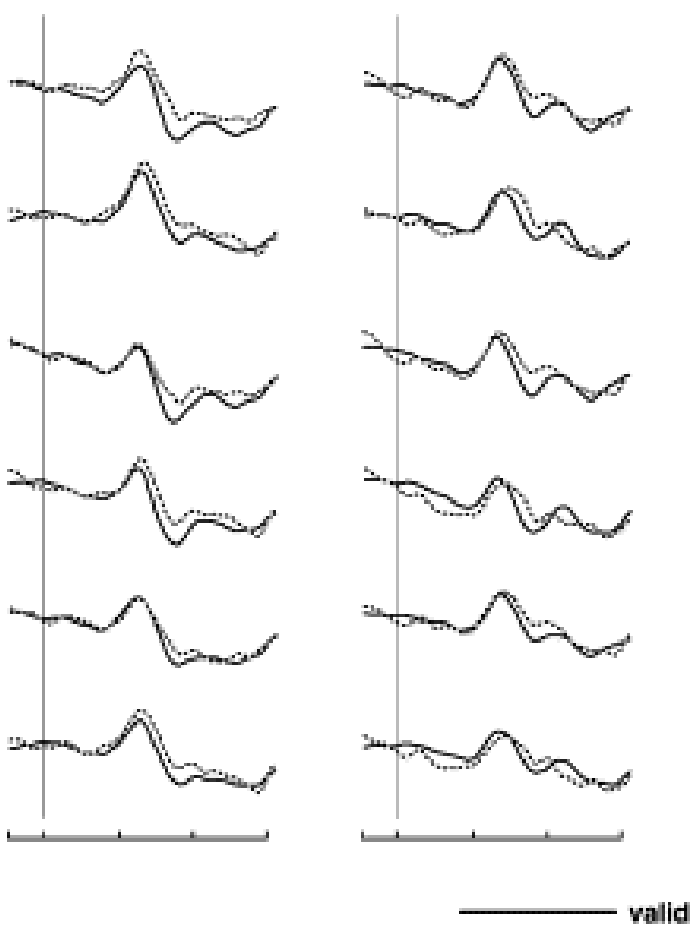

oL

CONTRALATERAL

IPSILATEAAL

Oค
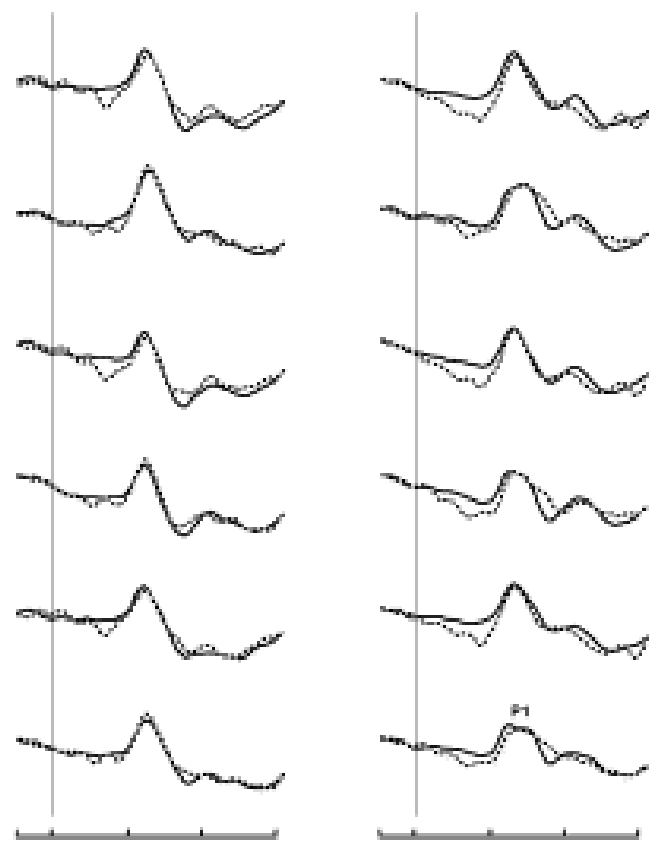

PL

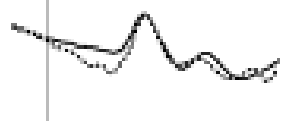

Ph

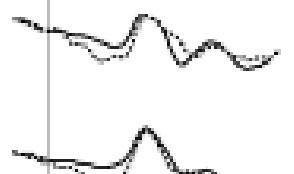

Ts

T6

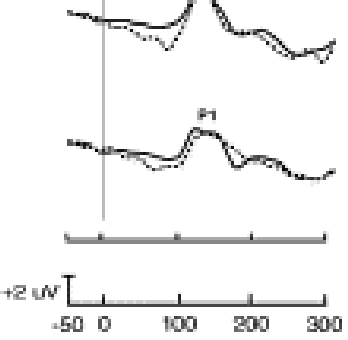

Figure 1. Grand-averaged ERPs for validly and invalidly targets at occipital, parietal and temporal electrodes ipsilateral and contralateral to the target location in the informative-peripheral cue condition. 


\section{UNINFORMATIVE-PERIPHERAL CUEING}

SOA 100

CONTRALATEAAL

IPSILATERAL

CONTRALATEAAL

SOA 300
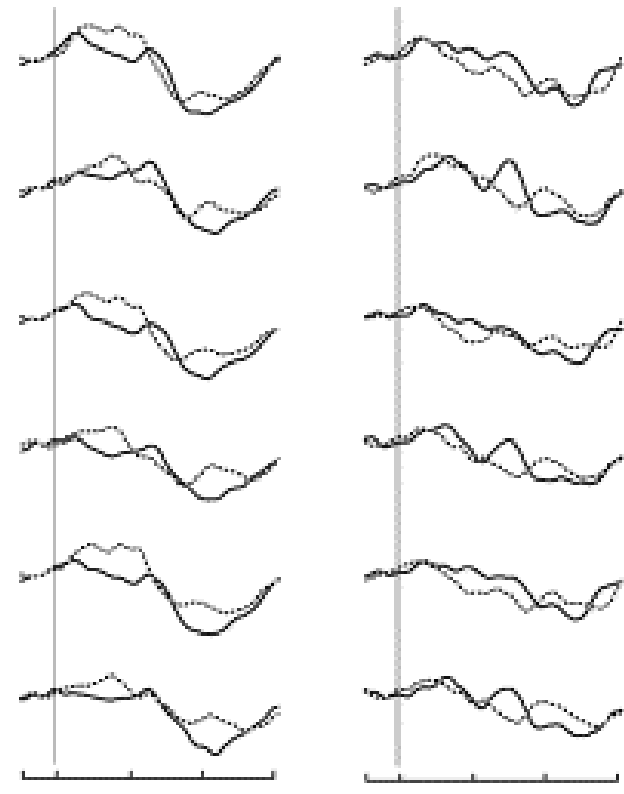

oL
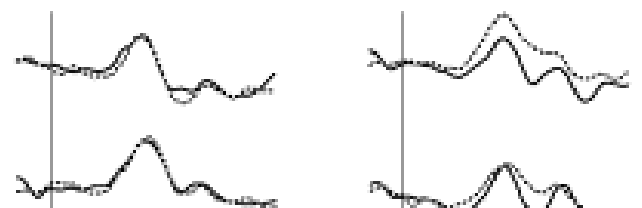

PL
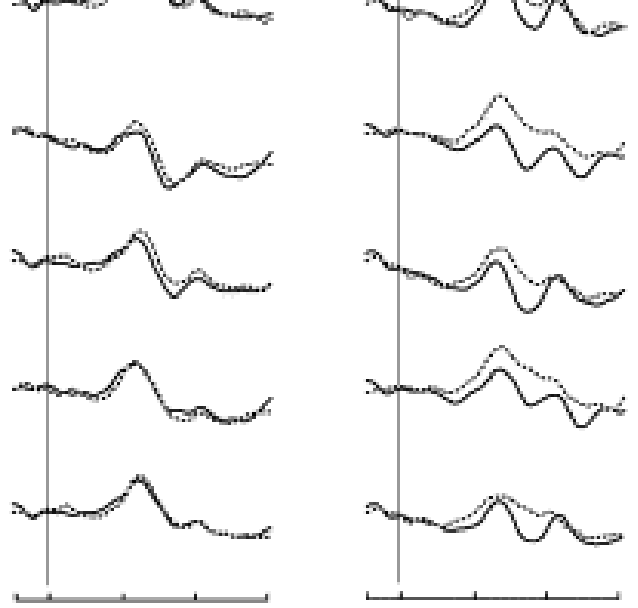

SOA 500

CONTRALATERAL

IPSILATEAAL

SOA 700
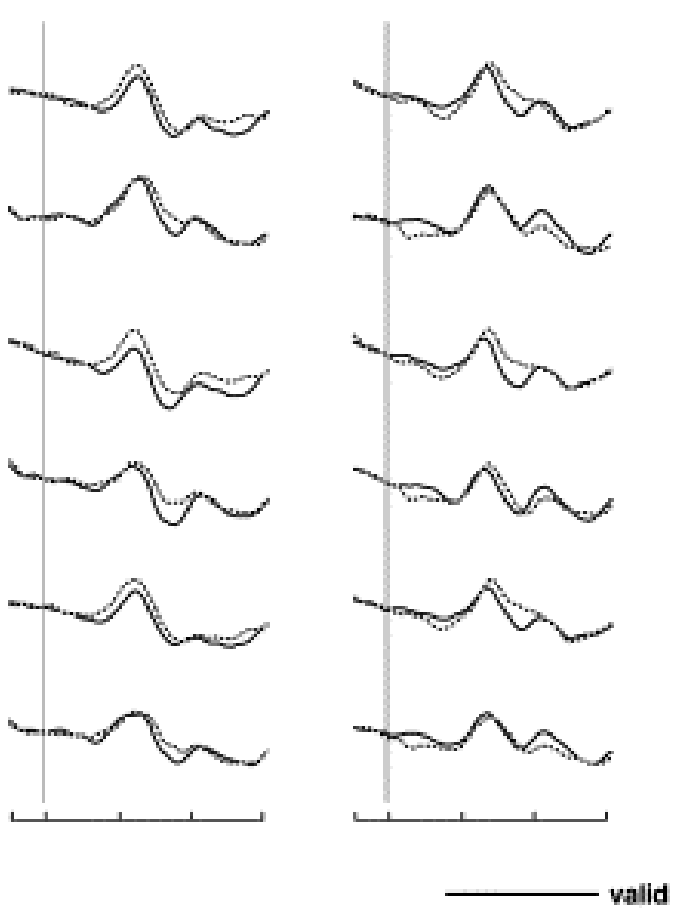

oL

CONTRALATEAAL

IPSILATEAAL
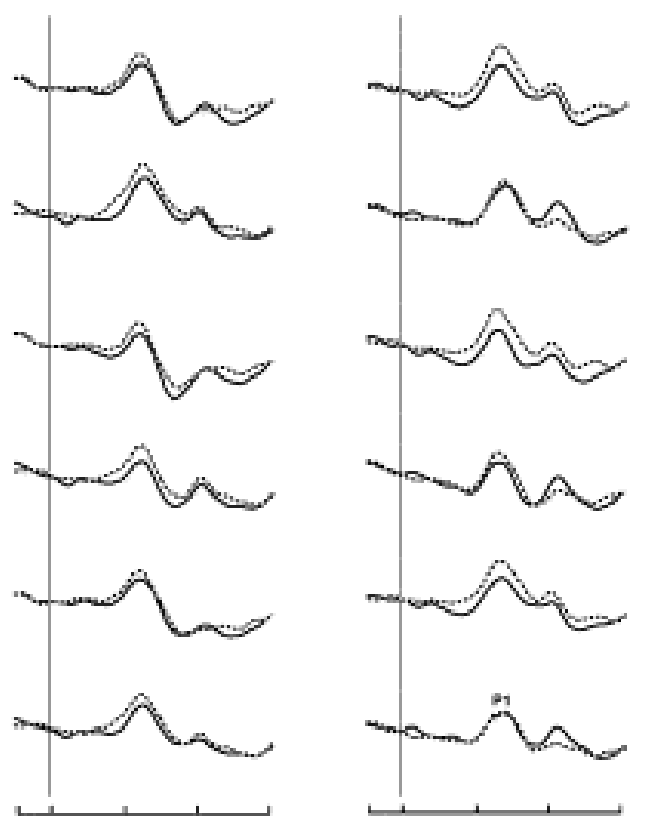

op

PL

Pด

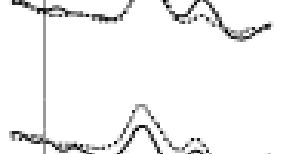

TS

To

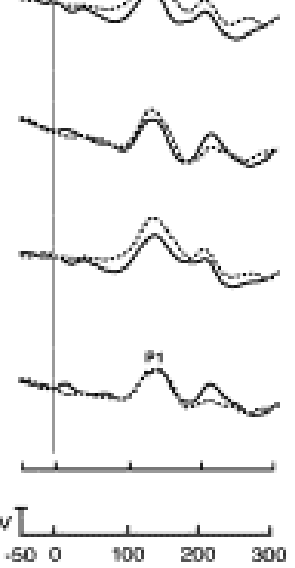

Figure 2. Grand-averaged ERPs for validly and invalidly targets at occipital, parietal and temporal electrodes ipsilateral and contralateral to the target location in the uninformative-peripheral cue condition. 
SOA 100

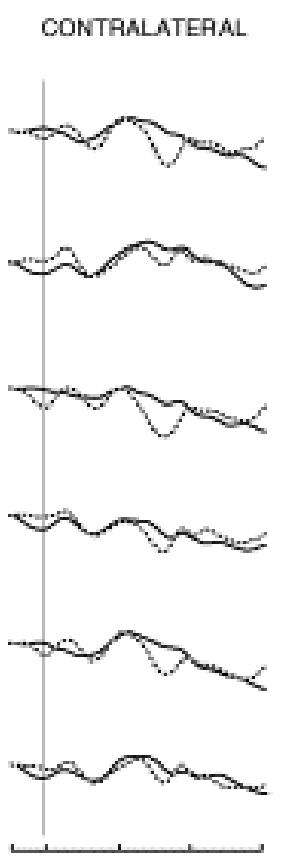

IPSILATERAL

CONTAALATERAL

IPSILATERAL
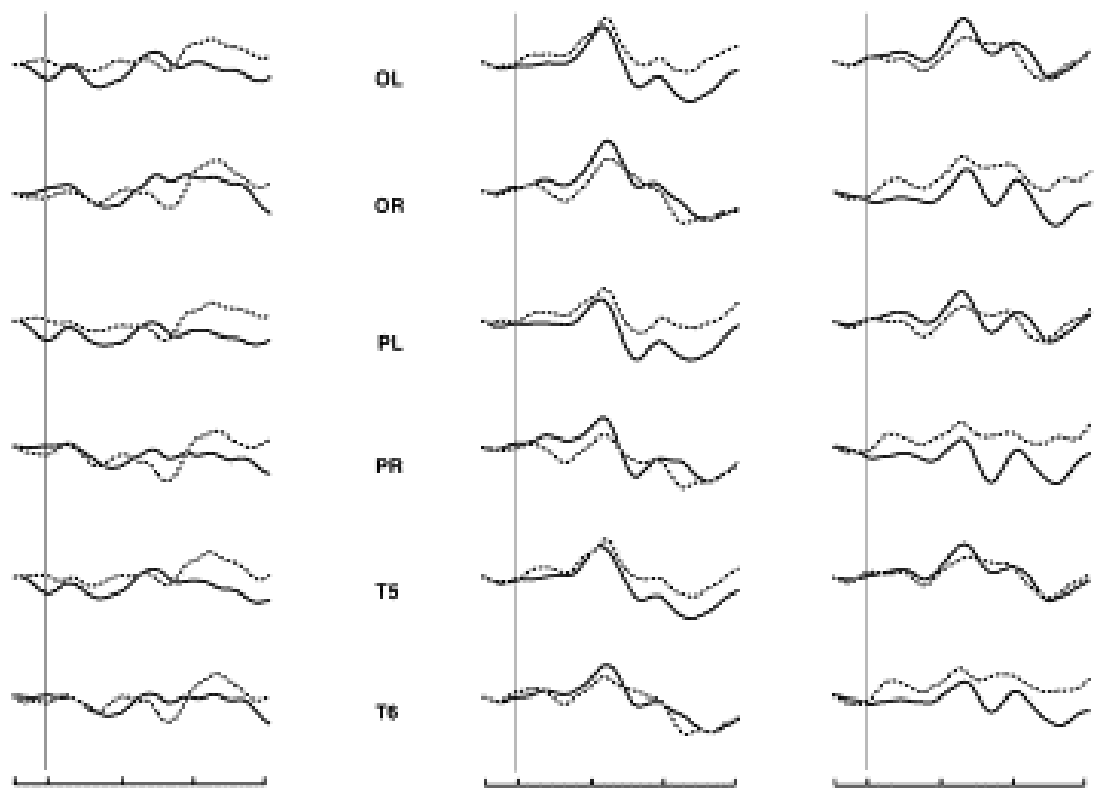

SOA 500

CONTRALATERAL

IPSILATERAL

SOA 700
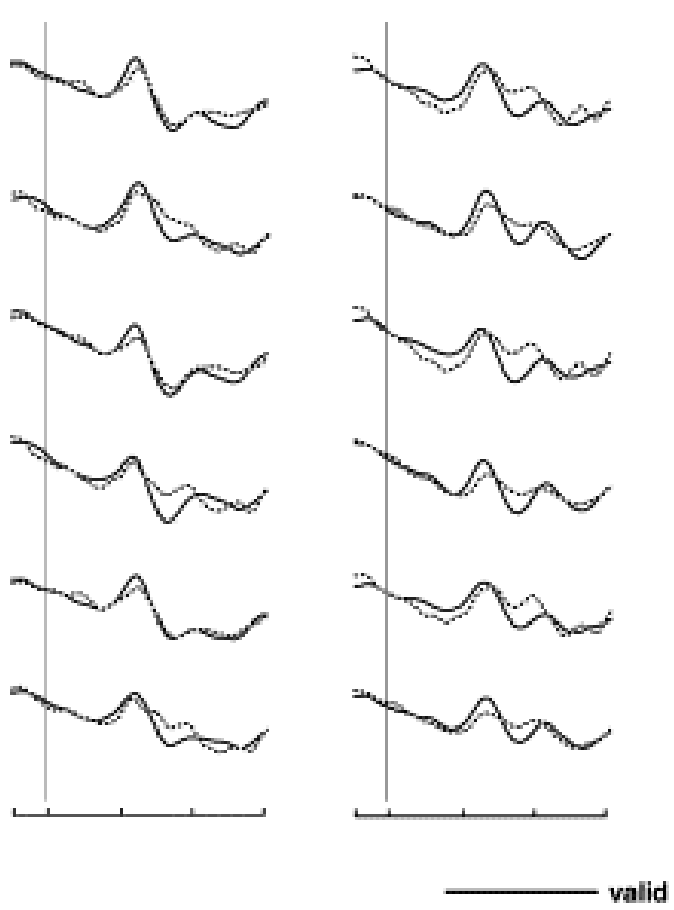

oL

CONTRALATERAL

IPSILATEAAL

op
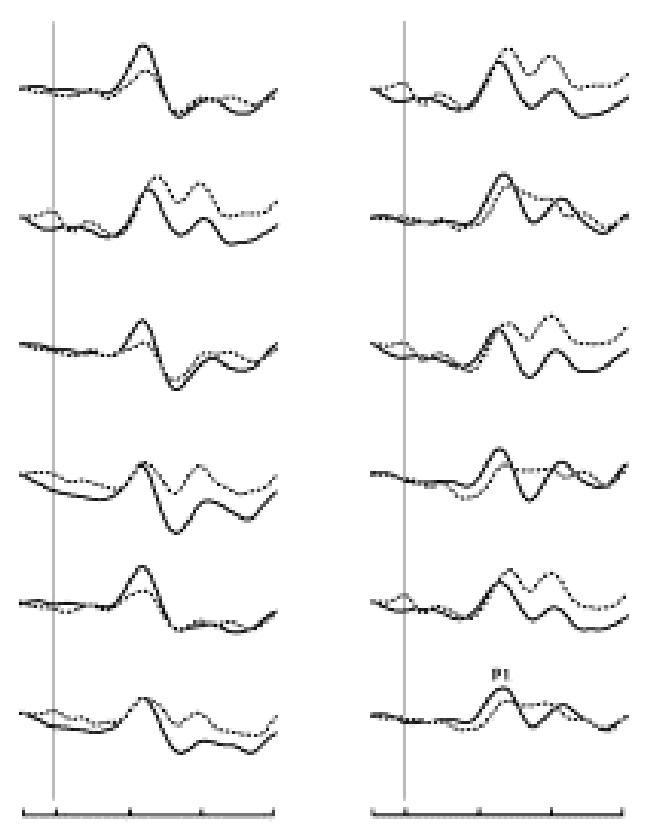

PL

Pค

TS

T6

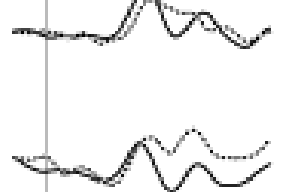

Invalid
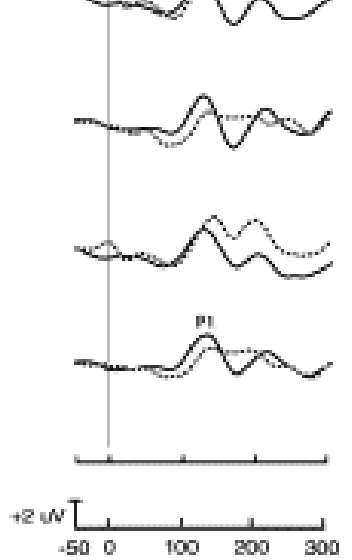

Figure 3. Grand-averaged ERPs for validly and invalidly targets at occipital, parietal and temporal electrodes ipsilateral and contralateral to the target location in the informative-central cue condition. 